\title{
Pressure-induced spin-state transition of iron in magnesiowüstite (Fe,Mg)O
}

\author{
I. Leonov \\ Theoretical Physics III, Center for Electronic Correlations and Magnetism, Institute of Physics, \\ University of Augsburg, 86135 Augsburg, Germany \\ and Materials Modeling and Development Laboratory, National University of Science and Technology “MISIS,” 119049 Moscow, Russia
}

A. V. Ponomareva

Materials Modeling and Development Laboratory, National University of Science and Technology “MISIS,” 119049 Moscow, Russia

R. Nazarov

Physics Division, Physical and Life Sciences Directorate, Lawrence Livermore National Laboratory, Livermore, California 94551, USA

I. A. Abrikosov

Department of Physics, Chemistry and Biology, Linköping University, SE-58183 Linköping, Sweden

(Received 7 April 2017; revised manuscript received 28 June 2017; published 17 August 2017)

\begin{abstract}
We present a detailed theoretical study of the electronic, magnetic, and structural properties of magnesiowüstite $\mathrm{Fe}_{1-x} \mathrm{Mg}_{x} \mathrm{O}$ with $x$ in the range between 0 and 0.875 using a fully charge self-consistent implementation of the density functional theory plus dynamical mean-field theory method. In particular, we compute the electronic structure and phase stability of the rocksalt $B 1$-structured $(\mathrm{Fe}, \mathrm{Mg}) \mathrm{O}$ at high pressures relevant for the Earth's lower mantle. We find that upon compression paramagnetic $(\mathrm{Fe}, \mathrm{Mg}) \mathrm{O}$ exhibits a spin-state transition of $\mathrm{Fe}^{2+}$ ions from a high-spin to low-spin (HS-LS) state which is accompanied by a collapse of local magnetic moments. The HS-LS transition results in a substantial drop in the lattice volume by about $4 \%-8 \%$, implying a complex interplay between electronic and lattice degrees of freedom. Our results reveal a strong sensitivity of the calculated transition pressure $P_{\mathrm{tr}}$. upon addition of $\mathrm{Mg}$. While, for Fe-rich magnesiowüstite with $\mathrm{Mg} x<0.5, P_{\mathrm{tr}}$ is about $80 \mathrm{GPa}$, for $\mathrm{Mg} x=0.75$ it drops to $52 \mathrm{GPa}$, i.e., by $35 \%$. This behavior is accompanied by a substantial change in the spin transition range from 50 to $140 \mathrm{GPa}$ in $\mathrm{FeO}$ to 30 to $90 \mathrm{GPa}$ for $x=0.75$. In addition, the calculated bulk modulus (in the HS state) is found to increase by $\sim 12 \%$ from $142 \mathrm{GPa}$ in $\mathrm{FeO}$ to $159 \mathrm{GPa}$ in $(\mathrm{Fe}, \mathrm{Mg}) \mathrm{O}$ with $\mathrm{Mg} x=0.875$. We find that the pressure-induced HS-LS transition has different consequences for the electronic properties of the Fe-rich and -poor $(\mathrm{Fe}, \mathrm{Mg}) \mathrm{O}$. For the Fe-rich $(\mathrm{Fe}, \mathrm{Mg}) \mathrm{O}$, the transition is found to be accompanied by a Mott insulator to a (semi)metal phase transition. In contrast to that, for $x>0.25$, $(\mathrm{Fe}, \mathrm{Mg}) \mathrm{O}$ remains insulating up to the highest studied pressures, implying a Mott-insulator to band-insulator phase transition at the HS-LS transformation.
\end{abstract}

DOI: 10.1103/PhysRevB.96.075136

\section{INTRODUCTION}

Magnesiowüstite $\left(\mathrm{Fe}_{1-x}, \mathrm{Mg}_{x}\right) \mathrm{O}$ is the second most abundant mineral in the Earth's interior and makes up some $20 \%$ of the total volume of Earth's lower mantle [1]. Therefore its high-pressure electronic properties, spin state of iron, and phase stability play an important role in the (geo-) physics, chemistry, and dynamics of the Earth's mantle. The high-pressure properties of $(\mathrm{Fe}, \mathrm{Mg}) \mathrm{O}$ have attracted much recent interest from both theoretical and experimental points of view. At ambient conditions, $(\mathrm{Fe}, \mathrm{Mg}) \mathrm{O}$ is known to exist as a solid solution between periclase $(\mathrm{MgO})$ and wüstite $(\mathrm{FeO})$. It has a rocksalt $B 1$ crystal structure with $\mathrm{Mg}^{2+}$ and high-spin $(S=2) \mathrm{Fe}^{2+}$ ions having octahedral environments. Furthermore, $(\mathrm{Fe}, \mathrm{Mg}) \mathrm{O}$ is likely to keep the $B 1$-type lattice structure throughout the Earth's lower-mantle conditions as suggested by recent x-ray diffraction measurements [2]. ( $\mathrm{Fe}, \mathrm{Mg}) \mathrm{O}$ comprises two end-member oxides with remarkably different electronic properties: $\mathrm{MgO}$ and $\mathrm{FeO} . \mathrm{MgO}$ is a band insulator with a $B 1$-type crystal structure stable up to $227 \mathrm{GPa}$ [3], whereas $\mathrm{FeO}$ is a prototypical Mott insulator with a complex interplay between electronic structure and lattice under pressure [4-13]. By changing the Mg content $x$, it seems therefore possible to tune a Mott- to band-insulator transition in (Fe, Mg)O [14-18].

High-pressure x-ray emission and Mössbauer spectroscopy experiments show that the $\mathrm{Fe}^{2+}$ ion of $\mathrm{FeO}$ and $(\mathrm{Fe}, \mathrm{Mg}) \mathrm{O}$ undergoes a high-spin (HS) to low-spin (LS) transition at pressures relevant for the Earth's lower mantle [2,5,11,12,19-31]. It has been confirmed that $\mathrm{FeO}$ makes a Mott-insulatorto-metal transition at about $70 \mathrm{GPa}$, retaining the $B 1$-type lattice structure at high temperature [11-13]. For $(\mathrm{Fe}, \mathrm{Mg}) \mathrm{O}$, these studies reveal that the transition pressure decreases upon the increase in the $\mathrm{Mg}$ content. In addition, they show that the HS-LS transition affects the electronic and elastic properties of $(\mathrm{Fe}, \mathrm{Mg}) \mathrm{O}$ and therefore has significant implications for the physics and chemistry of Earth. On the theoretical side, the electronic properties of $\mathrm{FeO}$ and $(\mathrm{Fe}, \mathrm{Mg}) \mathrm{O}$ have been intensively investigated employing bandstructure-based techniques [32-45]. These studies confirm a remarkable composition dependence of the pressure-induced spin-state transition of $\mathrm{Fe}^{2+}$ in $(\mathrm{Fe}, \mathrm{Mg}) \mathrm{O}$, showing, however, a broad scattering for the calculated transition pressures. Therefore the effects of temperature and composition on the spin-state transition pressure and broadness of the spin crossover have remained uncertain. All this makes a detailed 
study of the entire solid solution of $(\mathrm{Fe}, \mathrm{Mg}) \mathrm{O}$ essential for understanding its electronic state and magnetic properties.

These experimental and theoretical studies have led us to reinvestigate the properties of the $B 1$-type $(\mathrm{Fe}, \mathrm{Mg}) \mathrm{O}$ at high pressures employing a fully charge self-consistent implementation of the density functional plus dynamical meanfield theory method (DFT + DMFT) of strongly correlated electrons [46-49]. The DFT + DMFT method [50-53] allows one to capture all generic aspects of a pressure-induced Mottinsulator-to-metal phase transition (MIT), such as coherent quasiparticle behavior, the formation of the lower and upper Hubbard bands, and strong renormalization of the effective electron mass (reduced electron mobility) [54-66]. Most importantly, applications of DFT + DMFT have been shown to provide a good qualitative and even quantitative description of the electronic structure and phase stability of correlated materials, even in the vicinity of a MIT [67-71].

We employ DFT + DMFT to investigate the electronic structure, spin state of iron, and phase stability of paramagnetic $(\mathrm{Fe}, \mathrm{Mg}) \mathrm{O}$ at high pressure for a broad range of $\mathrm{Mg}$ compositions $x=0-0.875$, which have remained unexplored up to now. Our results reveal that $(\mathrm{Fe}, \mathrm{Mg}) \mathrm{O}$ exhibits a pressure-induced spin-state transition of $\mathrm{Fe}^{2+}$ ions from the HS to LS state which is accompanied by a collapse of local moments. Our results show a strong sensitivity of the electronic and lattice properties, transition pressure, and transition range of the HS-to-LS state crossover to Mg content $x$, indicating a complex interplay between electronic and lattice degrees of freedom. For Fe-rich (Fe,Mg)O, the HS-LS transition is found to be accompanied by a Mott-insulator to (semi)metal phase transition. In contrast to that, for $\mathrm{Mg}$ content above 0.25 , (Fe, $\mathrm{Mg}) \mathrm{O}$ remains insulating up to the highest studied pressures. This implies that the HS-LS transition is accompanied by a Mott-insulator to band-insulator phase transition for $x>0.25$. Our results for the electronic structure and lattice properties are in overall good agreement with experimental data.

\section{METHOD}

In this work, we provide a detailed theoretical study of the electronic structure, magnetic state, and phase stability of paramagnetic $B 1$-structured $(\mathrm{Fe}, \mathrm{Mg}) \mathrm{O}$ using a fully charge self-consistent implementation of the DFT + DMFT method [50-53,68-70]. We use this advanced theory to compute the high-pressure and -temperature properties of ( $\mathrm{Fe}, \mathrm{Mg}) \mathrm{O}$ as a function of $\mathrm{Mg}$ content $x$ in the range between 0 and 0.875 , i.e., above the percolation limit $(\sim 12 \% \mathrm{Fe})$ of the fcc lattice of $B 1$ type (Fe, Mg)O [72]. To this end, we calculate the total energy and (instantaneous) local magnetic moments $\sqrt{\left\langle m_{z}^{2}\right\rangle}$ of $B 1$-type (Fe,Mg)O as a function of lattice volume for different $\mathrm{Mg} x$ [73]. To model a chemical substitution $\mathrm{Fe} / \mathrm{Mg}$, we construct a supercell (with periodic boundary conditions) containing $8 \mathrm{f}$.u. of the host material $\mathrm{FeO}$ in which one to seven $\mathrm{Fe}$ ions were replaced with $\mathrm{Mg}$. The positions of the impurity atoms $(\mathrm{Mg} / \mathrm{Fe})$ were arranged to maximize the distance from each other [74] (Fe/ Mg atoms are uniformly distributed over the unit cell; that is, we neglect the possible formation of the $\mathrm{Fe} / \mathrm{Mg}$ clusters under pressure [75]). For simplicity, we neglect the local relaxation effects around the impurity $\mathrm{Mg} / \mathrm{Fe}$ atoms, as well as the possible formation of a site-selective Mott-insulating phase with coexisting (within a unit cell) HS and LS iron sites [76]. In order to evaluate pressure, we fit our total-energy results to the third-order Birch-Murnaghan equation of state [77] separately for the HS and LS volume regions. The HS-LS transition pressure and the corresponding drop in the lattice volume are determined from a Maxwell construction [as a common tangent to the calculated $E(V)$ curves]. The compressed phase is denoted by the relative volume with respect to the calculated equilibrium lattice volume as $v \equiv V / V_{0}$.

We employ the DFT + DMFT approach implemented within the plane-wave pseudopotentials [68-70] with the generalized gradient approximation in DFT [78,79]. For the partially filled $\mathrm{Fe} 3 d$ and $\mathrm{O} 2 p$ orbitals we construct a basis set of Wannier functions $[80,81]$ using the projection procedure on a local atomic-centered symmetry-constrained basis set as discussed in Refs. [82-84], with a window spanning both the $\mathrm{Fe} 3 d$ and $\mathrm{O} 2 p$ bands. We model a chemical disorder in (Fe,Mg)O by applying averaging of the Green's functions of the Fe sites [within the 8-f.u. supercell of $(\mathrm{Fe}, \mathrm{Mg}) \mathrm{O}$ ] because the Green's function is a self-averaging property of a random alloy [85]. We employ a single-site DFT + DMFT approach to treat the effects of electron correlation in the $\mathrm{Fe} 3 d$ shell, i.e. neglect the effect of spatial (nonlocal) correlations. To solve the realistic many-body problem, we employ the continuoustime hybridization-expansion (segment) quantum Monte Carlo algorithm [86]. The calculations are performed in the paramagnetic state at an electronic temperature $T=1160 \mathrm{~K}$. In accordance with previous studies of $\mathrm{FeO}$, we use the local Coulomb interaction $U=7 \mathrm{eV}$ and Hund's exchange $J=$ $0.89 \mathrm{eV}$ parameters for the Fe $3 d$ orbitals [13,60,62,69-71]. The $U$ and $J$ values are assumed to remain constant upon variation of the lattice volume. The Coulomb interaction is treated in the density-density approximation. The spin-orbit coupling is neglected in these calculations. We employ the fully localized double-counting correction, evaluated from the self-consistently determined local occupations, to account for the electronic interactions already described by DFT. The spectral functions were computed using the maximum-entropy method. The angle-resolved spectra were evaluated from analytic continuation of the self-energy using Padé approximants.

\section{RESULTS AND DISCUSSION}

As a starting point, we calculate the electronic structure, magnetic state, and phase stability of the 8-f.u. supercell of the $B 1$-structured paramagnetic phase of pure $\mathrm{FeO}(\mathrm{Mg} x=0)$. In Fig. 1 (left) we display our results for the total energy and (instantaneous) magnetic local moments $\sqrt{\left\langle m_{z}^{2}\right\rangle}$ computed within DFT + DMFT for different compressions of the lattice $\left(v \equiv V / V_{0}\right)$. Our results agree quantitatively well with those previously published in Refs. [69-71]. In particular, within the $B 1$ lattice structure of $\mathrm{FeO}$, a high-spin to low-spin transition is found to occur upon compression to $v<0.72$, i.e., above $\sim 73 \mathrm{GPa}$. The calculated bulk modulus $K_{0, T}$ for the low-pressure phase is $142 \mathrm{GPa}$; the (instantaneous) local magnetic moment $\sqrt{\left\langle m_{z}^{2}\right\rangle} \sim 3.7 \mu_{B}$, which corresponds to a fluctuating moment of $\mu_{z}=3.6 \mu_{B}$ [ $\mu_{z}$ is defined as an imaginary-time average of 

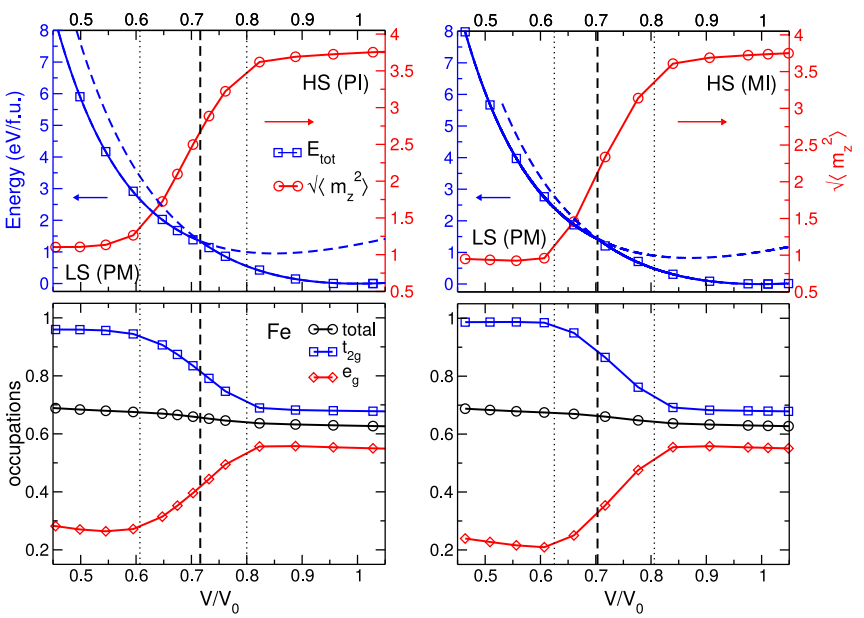

FIG. 1. Top: DFT + DMFT results for the total energy (blue) and instantaneous magnetic moments $\sqrt{\left\langle m_{z}^{2}\right\rangle}$ (red) of paramagnetic FeO (left) and $\left(\mathrm{Fe}_{0.75}, \mathrm{Mg}_{0.25}\right) \mathrm{O}$ (right) as a function of lattice volume. The thermodynamically unstable HS/LS EOS solutions (in the LS/HS state, respectively) are depicted by blue dashed lines. The HS-LS state transition is depicted by a vertical black dashed line. The HS-LS transition range (upper and lower onsets of the transition) is shown by two vertical dotted lines. Bottom: Fe $3 d$ and partial $t_{2 g}$ and $e_{g}$ occupations as a function of volume.

the local spin-spin correlation function $\chi(\tau)=\langle\hat{m}(\tau) \hat{m}(0)\rangle$ as $\mu_{z}=\left[1 / \beta \int_{0}^{\beta} d \tau \chi(\tau)\right]^{1 / 2}$, where $\tau$ is the imaginary time and $\beta$ is the inverse temperature $\beta=1 / k_{B} T$ ]. Our results show that the bulk modulus in the LS phase of $\mathrm{FeO}$ is substantially larger than that in the HS phase (142 GPa). In fact, for the LS state our estimate of $K_{0, T}$ is about $210 \mathrm{GPa}$ [87]. The HS-LS state transformation is accompanied by a Mott-insulator-to-metal phase transition [62] with a drop in the lattice volume by about 9\% at the MIT (from the lattice constant $a=7.6$ to 7.37 a.u.), implying a complex interplay between electronic and lattice degrees of freedom [69-71]. We also note that the calculated HS-LS transition pressure depends sensitively on the choice of the interaction parameters $U$ and $J$. In particular, for $\mathrm{FeO}$, it is about $55 \mathrm{GPa}$ for $U=5 \mathrm{eV}$ and $\sim 80 \mathrm{GPa}$ for $U=8 \mathrm{eV}[69,70]$.

Under pressure, our results indicate a substantial charge transfer in the $\mathrm{Fe}^{2+} 3 d$ shell between the $t_{2 g}$ and $e_{g}$ states. Namely, the occupancy of the $t_{2 g}$ orbitals gradually increases, resulting in an (almost) completely occupied state (with a $t_{2 g}$ occupation of about 0.95 ). In contrast to that, the $e_{g}$ orbitals are strongly depopulated (their occupation is below 0.3 ), while the total $\mathrm{Fe} 3 d$ occupancy remains essentially unchanged with pressure. Our results for the high-pressure electronic, magnetic, and lattice properties of $\mathrm{FeO}$, e.g., that above $\sim 73 \mathrm{GPa}$ the $B 1$-structured $\mathrm{FeO}$ undergoes a HS-toLS transition that is accompanied by a MIT and collapse of the lattice volume, are in overall good agreement with recent experimental data [11-13]. Moreover, in accordance with previous studies, our calculations clearly indicate the crucial importance of electronic correlations to determine the high-pressure properties of $\mathrm{FeO}$ [13,69-71]. In particular, treating the $\mathrm{LS}$ metallic phase of $\mathrm{FeO}$ within the nonmagnetic generalized gradient approximation cannot explain a relatively
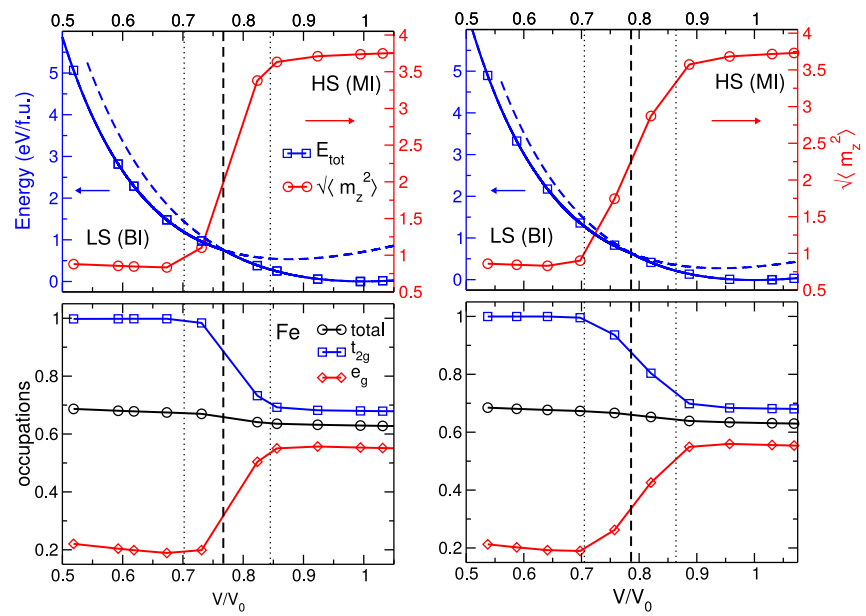

FIG. 2. Top: Results for total energy (blue) and instantaneous moments $\sqrt{\left\langle m_{z}^{2}\right\rangle}$ (red) of magnesiowüstite with Mg content $x=0.5$ (left) and 0.75 (right) calculated by DFT + DMFT for different lattice volumes. Bottom: The Fe $3 d$ and partial $t_{2 g}$ and $e_{g}$ occupations as a function of volume.

large band renormalization $m^{*} / m \sim 2$ above the HS-LS transition and predicts the metallic phase is stable at ambient pressure with respect to the Mott-insulating phase (evaluated within DFT + DMFT). In this case, the Mott-insulating phase of $\mathrm{FeO}$ appears to be stable only upon expansion of the lattice volume.

Next, we compute the electronic structure and lattice properties of $(\mathrm{Fe}, \mathrm{Mg}) \mathrm{O}$ as a function of $\mathrm{Mg}$ content $x$ under pressures relevant to the Earth's lower-mantle conditions. In Figs. 1 and 2 we present our results for the total energy and local moments $\sqrt{\left\langle m_{z}^{2}\right\rangle}$ of the $B 1$-structured $(\mathrm{Fe}, \mathrm{Mg}) \mathrm{O}$ calculated within DFT + DMFT for different $v \equiv V / V_{0}$. Our results for the bulk modulus and equilibrium lattice volume evaluated from the DFT + DMFT total-energy calculations are summarized in Table I. At ambient pressure, for all $x$ we obtain a Mott-insulating solution with a large $d-d$ energy gap of about $2 \mathrm{eV}$, in accordance with previous studies [60,71]. Our results for the Fe $t_{2 g}$ and $e_{g}$ orbital occupations are about 0.65 and 0.55 , respectively, near half filling, implying the HS $(S=2)$ state of $\mathrm{Fe}^{2+}$ ions. In addition, similar to $\mathrm{FeO}$, the instantaneous local moment is about $3.7 \mu_{B}$ (fluctuating moment of $3.6 \mu_{B}$ ). The Fe $3 d$ electrons are localized, as seen from our result for the local spin-spin correlation function $\chi(\tau)=\langle\hat{m}(\tau) \hat{m}(0)\rangle$ shown in Fig. 3 (where $\tau$ is the imaginary time). In fact, $\chi(\tau)$ is seen to be almost constant and close to its maximal value for the partial Fe $3 d$ states (i.e., to unity), indicating localization of $3 d$ electrons at ambient pressure. In contrast to that a strong pressure-induced suppression of $\chi(\tau)$ for large $\tau$, i.e., the absence of long-living local magnetic moments, indicates a crossover to a delocalized state. We also point out the crucial importance of the effects of electron correlation to determine the electronic properties of $(\mathrm{Fe}, \mathrm{Mg}) \mathrm{O}$.

Upon compression, our calculations show that $(\mathrm{Fe}, \mathrm{Mg}) \mathrm{O}$ compounds undergo a HS-LS phase transition, with a collapse of the local moments to a LS state [60,71]. The LS state is characterized by a fluctuating magnetic moment $\mu_{z}$ which is below $\sim 0.2 \mu_{B}-0.4 \mu_{B}$ for compression $v \leqslant 0.6-0.7$, i.e., 
TABLE I. Calculated structural parameters for the paramagnetic $B 1$ phase of $(\mathrm{Fe}, \mathrm{Mg}) \mathrm{O}$ for different $\mathrm{Mg}$ content $x$. $V_{0}$ is ambient pressure volume. $K_{0, T}$ is the bulk modulus for the HS and LS phases; $K^{\prime} \equiv d K / d P$ is fixed to 4.1 for all $\mathrm{Mg}$ compositions. $P_{\text {tr. }}$ is the HS-LS transition pressure. $|\Delta V|$ and $\Delta V / V$ are absolute and relative changes in the lattice volume at the HS-LS transition.

\begin{tabular}{|c|c|c|c|c|c|c|}
\hline $\operatorname{Mg}_{x}$ & $V_{0}$ (a.u. ${ }^{3} /$ f.u. $)$ & $K_{0, T}^{H S}(\mathrm{GPa})$ & $K_{0, T}^{L S}(\mathrm{GPa})$ & $P_{\text {tr. }}(\mathrm{GPa})$ & $|\Delta V|$ (a.u. ${ }^{3} /$ f.u. $)$ & $\Delta V / V(\%)$ \\
\hline 0 & 144.1 & 142 & 210 & 73 & 10.2 & 9 \\
\hline 0.125 & 143.1 & 139 & 205 & 82 & 8.3 & 8 \\
\hline 0.25 & 141.3 & 137 & 201 & 83 & 7.1 & 7 \\
\hline 0.375 & 139.5 & 138 & 213 & 77 & 7.2 & 7 \\
\hline 0.5 & 138.6 & 139 & 200 & 49 & 8.6 & 8 \\
\hline 0.625 & 135.5 & 142 & 185 & 61 & 5.2 & 5 \\
\hline 0.75 & 133.8 & 151 & 169 & 52 & 4.7 & 4 \\
\hline 0.875 & 132.9 & 159 & 158 & 21 & 5.1 & 4 \\
\hline
\end{tabular}

above $\sim 150 \mathrm{GPa}$. Interestingly, in the same pressure range, the LS FeO has a fluctuating moment of $\sim 0.7 \mu_{B}$, i.e., remarkably higher than that in the $\mathrm{LS}$ state of $(\mathrm{Fe}, \mathrm{Mg}) \mathrm{O}$. Like for $\mathrm{FeO}$, we observe a substantial redistribution of charge between the Fe $t_{2 g}$ and $e_{g}$ orbitals within the Fe $3 d$ shell caused by applied pressure. Above the HS-LS transition, it leads to an (almost) complete occupation of the Fe $t_{2 g}$ states, while the Fe $e_{g}$ states are strongly depopulated (with occupancy below 0.2-0.3).

The HS-LS spin-state transition is found to be accompanied by a substantial drop in lattice volume of $\sim 4 \%-8 \%$ (see
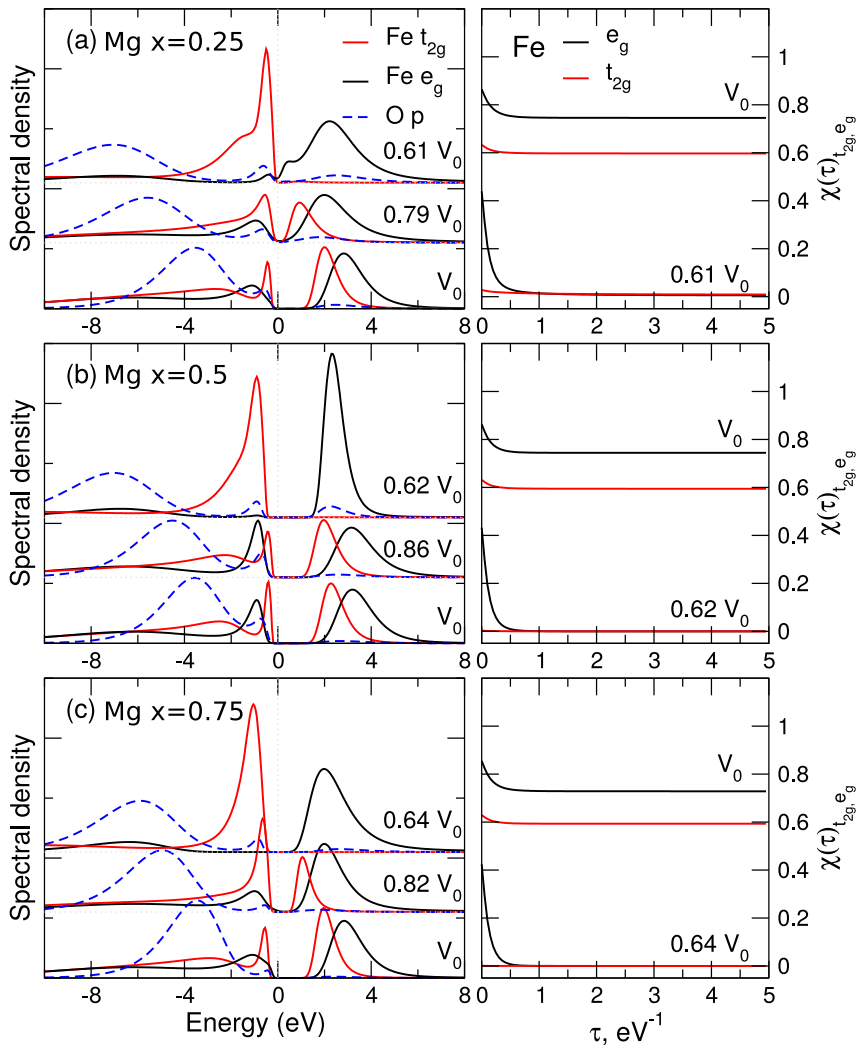

FIG. 3. Left: Partial Fe $t_{2 g}$ and $e_{g}$ and $\mathrm{O} 2 p$ spectral functions of magnesiowüstite with $\mathrm{Mg}$ content (a) $x=0.25$, (b) 0.5 , and (c) 0.75 calculated by DFT + DMFT for different lattice volumes. Right: Local spin-spin correlation function $\chi(\tau)$ calculated by DFT + DMFT as a function of volume. The intraorbital $t_{2 g}$ and $e_{g}$ contributions are shown.
Table I). We note, however, that these values should be considered an upper-bound estimate because we neglect multiple intermediate-phase transitions when fitting the totalenergy result to the third-order Birch-Murnaghan equation of states [77]. The structural change takes place upon a compression of the lattice volume to $v \sim 0.7-0.8$. Our results for the calculated transition pressures are about 83 and $52 \mathrm{GPa}$ for $\mathrm{Mg}$ contents of $x=0.25$ and 0.75 , respectively. This implies that the electronic and structural properties of (Fe, $\mathrm{Mg}$ ) O are strongly sensitive to the addition of $\mathrm{Mg}$. While for Fe-rich $(\mathrm{Fe}, \mathrm{Mg}) \mathrm{O}$, for $x<0.5$, the calculated transition pressure exhibits a rather weak variation at around $80 \mathrm{GPa}$, for the Fe-poor compounds the HS-LS transition pressure drops substantially, to $52 \mathrm{GPa}$, i.e., by $\sim 35 \%$, for $x=0.75$. We also note a substantial increase from $\sim 140$ to $160 \mathrm{GPa}$, i.e., by about $12 \%$, of the calculated bulk modulus in $\mathrm{HS}(\mathrm{Fe}, \mathrm{Mg}) \mathrm{O}$ for $x>0.5$. This behavior is accompanied by a gradual decrease in the equilibrium lattice volume of $(\mathrm{Fe}, \mathrm{Mg}) \mathrm{O}$, as shown in Fig. 4. In addition, we obtain a substantial change in the HS-LS transition range, from $\sim 50-140 \mathrm{GPa}$ in $\mathrm{FeO}$ to $30-88 \mathrm{GPa}$ in (Fe, Mg)O with $\mathrm{Mg} x=0.75$. This indicates that the HS-LS transition width decreases with $\operatorname{Mg} x$, in agreement with recent experiments $[29,88]$.

Our results for the electronic properties, equilibrium volume, and phase stability of $(\mathrm{Fe}, \mathrm{Mg}) \mathrm{O}$ with $\mathrm{Mg} x=$ 0-0.875 calculated within DFT + DMFT agree well with available experimental data [22,27,29,30,88]. Overall, they are (qualitatively) consistent with the high-pressure behavior of pure FeO. Moreover, we observe a substantial change in the behavior of the Fe $3 d$ electrons, which exhibit a crossover from a localized to itinerant magnetic behavior under pressure, implying delocalization of $3 d$ electrons [69,70]. Interestingly, similar to $\mathrm{FeO}$, the calculated bulk moduli of ( $\mathrm{Fe}, \mathrm{Mg}) \mathrm{O}$ exhibit a sharp increase at the HS-LS transition, except for $\mathrm{Mg}$ $x=0.875$, where $K_{0, T}$ remains essentially unchanged $(\sim 158-$ $159 \mathrm{GPa})$ at the HS-LS transition. Furthermore, we note that $\mathrm{Fe}$-rich and -poor (Fe,Mg)O compounds, although both exhibit a HS-LS transition, show remarkably different electronic properties at high pressures. In particular, for the Fe-rich (Fe,Mg)O compounds with $\mathrm{Mg}$ content $x \leqslant 0.25$, a HS-LS transition in the $B 1$-type structure results in metallization, i.e., a Mott-insulator to (semi)metal phase transition. In fact, (Fe,Mg)O with $x=0.25$ shows bad metal behavior at high pressures, as shown in Fig. 3(a). In addition, our results for the k-resolved spectral function of $(\mathrm{Fe}, \mathrm{Mg}) \mathrm{O}$ with $x=0.25$ 


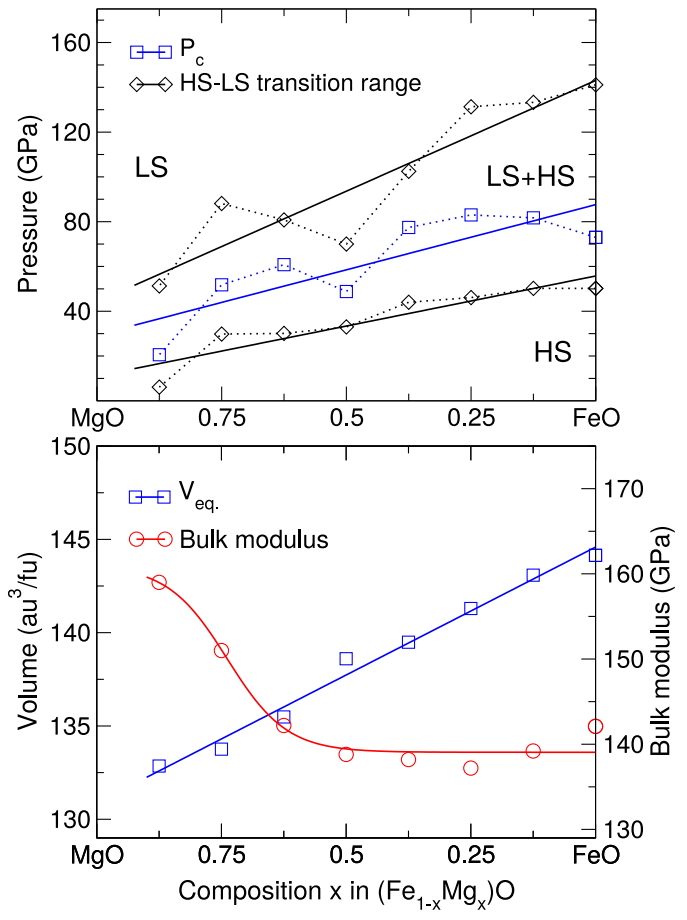

FIG. 4. Results for the HS-LS transition pressure and transition pressure range (upper and lower onsets of the transition; top) and the equilibrium lattice volume and bulk modulus (bottom) of $(\mathrm{Fe}, \mathrm{Mg}) \mathrm{O}$ as a function of $\mathrm{Mg} x$ calculated by DFT + DMFT at $T=1160 \mathrm{~K}$.

(see Fig. 5) show a semimetallic behavior with a substantial broadening of the electronic states near the Fermi level due to the effect of electron-electron correlations. In contrast to that, for $\mathrm{Fe}$-poor $(\mathrm{Fe}, \mathrm{Mg}) \mathrm{O}$ with $\mathrm{Mg} x>0.25$ the high-pressure $\mathrm{LS}$ phase is an insulator. Moreover, for magnesiowüstite with $\mathrm{Mg}$ $x>0.25$, the energy gap (as partly seen in Fig. 3) is found to increase upon compression above the HS-LS transition. Our analysis of the high-pressure behavior of the self-energy of the Fe-poor ( $\mathrm{Fe}, \mathrm{Mg}) \mathrm{O}$ compounds suggests that the HS-LS transition is accompanied by a Mott-insulator to band-insulator phase transition [15-18]. Indeed, in the latter case, e.g., for $(\mathrm{Fe}, \mathrm{Mg}) \mathrm{O}$ with $x=0.75$, the electronic states are seen to be highly coherent, revealing no finite-time broadening effects in electronic spectrum that are usually caused by the effects of electron-electron correlations. This implies that the effects of dynamical electronic correlations are weak for the $\mathrm{Fe}$-poor $(\mathrm{Fe}, \mathrm{Mg}) \mathrm{O}$, suggesting the importance of the effects of crystal-field splitting and their enhancement caused by static correlations.

In Fig. 4 we summarize our results for the behavior of $(\mathrm{Fe}, \mathrm{Mg}) \mathrm{O}$ as a function of $\mathrm{Mg}$ content $x$ calculated within DFT + DMFT. We observe that $(\mathrm{Fe}, \mathrm{Mg}) \mathrm{O}$ compounds show a HS-LS phase transition, with a collapse of the local magnetic moment to a LS state. We find that the HS-LS transition pressure decreases upon addition of $\mathrm{Mg}$, in agreement with experimental data $[22,29,30]$. This implies that the addition of $\mathrm{FeO}$ in $\mathrm{MgO}$ results in stabilization of the HS state of $\mathrm{Fe}^{2+}$ to higher pressures. This behavior is accompanied by a substantial increase in the equilibrium volume of the HS phase of $(\mathrm{Fe}, \mathrm{Mg}) \mathrm{O}$ by $\sim 9 \%$ upon moving from $\mathrm{MgO}$ to $\mathrm{FeO}$. For Fe-rich $(\mathrm{Fe}, \mathrm{Mg}) \mathrm{O}$, the ambient-pressure bulk modulus

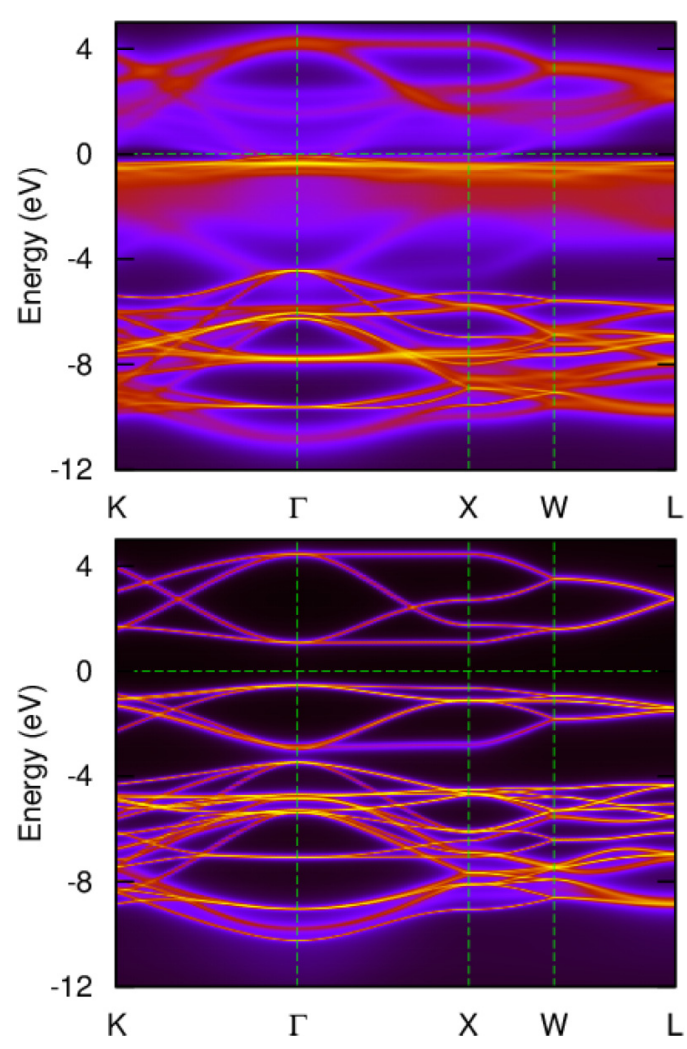

FIG. 5. The k-resolved Fe $3 d$ and $\mathrm{O} 2 p$ spectral function of paramagnetic high-pressure phases of $(\mathrm{Fe}, \mathrm{Mg}) \mathrm{O}$ as obtained by DFT + DMFT. Top: Results for the LS phase of $(\mathrm{Fe}, \mathrm{Mg}) \mathrm{O}$ with $\mathrm{Mg}$ content $x=0.25$ and lattice volume $v \sim 0.61$. Bottom: Results for the LS phase with $x=0.75$ and lattice volume compression of $\sim 0.64$.

$K_{0, T}$ shows a rather weak variation (about $140 \mathrm{GPa}$ ) with $\mathrm{Mg} x$. For the Fe-poor compounds, $K_{0, T}$ is found to increase substantially by $\sim 12 \%$ for $\mathrm{Mg} x>0.5$. We note that the addition of $\mathrm{Mg}$ can be interpreted as an effective chemical pressure acting on the high-spin $\mathrm{Fe}^{2+}$ ion. Indeed, our results indicate that this leads to an effective decrease in the bandwidth and increase in the $t_{2 g}-e_{g}$ crystal-field splitting of the Fe $3 d$ states (here we do not consider the effects of percolation since our calculations were performed above the percolation limit of $\sim 12 \% \mathrm{Fe}$ ). In addition, our DFT + DMFT calculations show a monotonous decrease of the equilibrium lattice volume upon addition of Mg. This may result in a HS Mott-insulator to LS band-insulator phase transition without metallization upon the increasing of the crystal-field splitting caused by applied pressures, in qualitative agreement with the generic phase diagram of a HS-LS transition in the two-orbital Hubbard model [89]. Our results for the $B 1$-structured $(\mathrm{Fe}, \mathrm{Mg}) \mathrm{O}$ provide a unified picture of the HS-LS transition in magnesiowüstite. While the $\mathrm{Fe}$-rich $(\mathrm{Fe}, \mathrm{Mg}) \mathrm{O}$ exhibit a rather weak variation of the electronic structure and lattice properties, the properties of the $\mathrm{Fe}$-poor compounds are remarkably different. It appears to be due to a more local nature of magnetic interactions of the $\mathrm{Fe}^{2+}$ ion in the $\mathrm{Fe}$-poor compounds. Indeed, the contribution of the Fe-Fe interatomic exchange interaction which tends to stabilize the HS state to much higher pressures is much weaker (or even absent) in $\mathrm{Fe}$-poor $(\mathrm{Fe}, \mathrm{Mg}) \mathrm{O}$. This suggests 
the importance of percolation effects for understanding the properties of $\mathrm{Fe}$-poor $(\mathrm{Fe}, \mathrm{Mg}) \mathrm{O}$.

In conclusion, we have determined the electronic properties, magnetic state, and phase stability of paramagnetic $B$ 1-structured magnesiowüstite $\mathrm{Fe}_{1-x} \mathrm{Mg}_{x} \mathrm{O}$ for $\mathrm{Mg}$ content $x$ in the range between 0 and 0.875 using DFT + DMFT. We computed the electronic structure and phase stability of the rocksalt $B 1$-structured $(\mathrm{Fe}, \mathrm{Mg}) \mathrm{O}$ at high pressures and temperatures relevant for the Earth's lower-mantle conditions. Upon compression paramagnetic (Fe, Mg)O exhibits a HSLS transition of $\mathrm{Fe}^{2+}$ ions which is accompanied by a simultaneous collapse of local moments. Our results reveal strong sensitivity of the calculated transition pressure $P_{\text {tr. }}$. upon addition of $\mathrm{Mg}$. While for Fe-rich magnesiowüstite $P_{\text {tr. }}$ has a rather weak variation and is about $80 \mathrm{GPa}$ for $x<0.5$, for the Fe-poor case it drops to about $21 \mathrm{GPa}$ for $x=0.875$. In agreement with experiment, this behavior is accompanied by an increase in the calculated bulk modulus in the HS phase of $(\mathrm{Fe}, \mathrm{Mg}) \mathrm{O}$ by about $12 \%$ for $\mathrm{Mg} x>0.5$. Moreover, the equilibrium lattice volume of (Fe, $\mathrm{Mg}) \mathrm{O}$ shows a substantial monotonous decrease with $\mathrm{Mg} x$. This suggests that the addition of $\mathrm{Mg}$ can be interpreted as an effective chemical pressure acting on the high-spin $\mathrm{Fe}^{2+}$ ion. Moreover, we found that the addition of $\mathrm{FeO}$ in $\mathrm{MgO}$ stabilizes the high-spin state to higher pressures. For the Fe-rich $(\mathrm{Fe}, \mathrm{Mg}) \mathrm{O}$, the transition is found to be accompanied by a Mott-insulator to (semi)metal phase transition. In contrast to that, for $\mathrm{Mg}$ content above $x>0.25$ (Fe, $\mathrm{Mg}$ )O remains insulating up to the highest studied pressures. Our results suggest that for $x>0.25$ the HS-LS transition is accompanied by a Mott-insulator to band-insulator phase transition. The lattice volume is found to collapse by about $4 \%-8 \%$ at the HS-LS transition, implying a complex interplay between electronic and lattice degrees of freedom. Our results indicate that for the $\mathrm{Mg}$ compositions relevant for the Earth's interior, i.e., $\mathrm{Mg} x=0.7-0.9$, the $\mathrm{Fe}^{2+}$ ion of $(\mathrm{Fe}, \mathrm{Mg}) \mathrm{O}$ is in a LS state throughout most of the Earth's lower mantle [30]. We point out the importance of further theoretical and experimental investigations of the behavior of $(\mathrm{Fe}, \mathrm{Mg}) \mathrm{O}$ at high pressures and temperatures, e.g., studying the effect of short-range ordering and formation of $\mathrm{Fe}$ clusters [75] and possible decomposition of (Fe, Mg)O [27,90], for a better understanding of the Earth's lower mantle and outer core.

\section{ACKNOWLEDGMENTS}

We thank D. Vollhardt, G. Kh. Rozenberg, and L. Dubrovinsky for valuable discussions. I.L. acknowledges support from the Deutsche Forschungsgemeinschaft through Transregio TRR 80 and the Ministry of Education and Science of the Russian Federation in the framework of the Increase Competitiveness Program of NUST MISIS (K3-2016-027), implemented by a governmental decree dated 16 March 2013, N 211. A.V.P. is grateful to the Russian Foundation for Basic Researches (Grant No. 16-02-00797) for financial support. I.A.A. acknowledges Swedish Research Council (VR) Grant No. 2015-04391 and the Swedish Government Strategic Research Area in Materials Science on Functional Materials at Linkoping University (Faculty Grant SFO-Mat-LiU No. 2009 00971).
[1] J.-F. Lin, S. Speziale, Z. Mao, and H. Marquardt, Rev. Geophys. 51, 244 (2013).

[2] J.-F. Lin, D. L. Heinz, H.-K. Mao, R. J. Hemley, J. M. Devine, J. Li, and G. Shen, Proc. Natl. Acad. Sci. USA 100, 4405 (2003).

[3] T. S. Duffy, R. J. Hemley, and H.-K. Mao, Phys. Rev. Lett. 74, 1371 (1995).

[4] E. Knittle and R. Jeanloz, J. Geophys. Res. 96, 16169 (1991).

[5] M. P. Pasternak, R. D. Taylor, R. Jeanloz, X. Li, J. H. Nguyen, and C. A. McCammon, Phys. Rev. Lett. 79, 5046 (1997).

[6] J. Badro, V. V. Struzhkin, J. Shu, R. J. Hemley, H.-k. Mao, C.-c. Kao, J.-P. Rueff, and G. Shen, Phys. Rev. Lett. 83, 4101 (1999).

[7] A. P. Kantor, S. D. Jacobsen, I. Y. Kantor, L. S. Dubrovinsky, C. A. McCammon, H. J. Reichmann, and I. N. Goncharenko, Phys. Rev. Lett. 93, 215502 (2004).

[8] M. Murakami, K. Hirose, S. Ono, T. Tsuchiya, M. Isshiki, and T. Watanuki, Phys. Earth Planet. Inter. 146, 273 (2004).

[9] Y. Fei and H.-K. Mao, Science 266, 1678 (1994).

[10] H. Ozawa, F. Takahashi, K. Hirose, Y. Ohishi, and N. Hirao, Science 334, 792 (2011).

[11] R. A. Fischer, A. J. Campbell, O. T. Lord, G. A. Shofner, P. Dera, and V. B. Prakapenka, Geophys. Res. Lett. 38, L24301 (2011).

[12] R. A. Fischer, A. J. Campbell, G. A. Shofner, O. T. Lord, P. Dera, and V. B. Prakapenka, Earth Planet. Sci. Lett. 304, 496 (2011).
[13] K. Ohta, R. E. Cohen, K. Hirose, K. Haule, K. Shimizu, and Y. Ohishi, Phys. Rev. Lett. 108, 026403 (2012).

[14] A. Fuhrmann, D. Heilmann, and H. Monien, Phys. Rev. B 73, 245118 (2006).

[15] S. S. Kancharla and S. Okamoto, Phys. Rev. B 75, 193103 (2007).

[16] P. Werner and A. J. Millis, Phys. Rev. Lett. 99, 126405 (2007).

[17] M. Sentef, J. Kuneš, P. Werner, and A. P. Kampf, Phys. Rev. B 80, 155116 (2009).

[18] H. Lee, Y.-Z. Zhang, H. O. Jeschke, and R. Valentí, Phys. Rev. B 89, 035139 (2014).

[19] E. Knittle, R. Jeanloz, A. C. Mitchell, and W. J. Nellis, Solid State Commun. 59, 513 (1986).

[20] J. Badro, V. V. Struzhkin, J. Shu, R. J. Hemley, H. K. Mao, J. P. Rueff, and C. C. Kao, Science 300, 789 (2003).

[21] A. F. Goncharov, V. V. Struzhkin, and S. D. Jacobsen, Science 312, 1205 (2006).

[22] J.-F. Lin, V. V. Struzhkin, S. D. Jacobsen, M. Hu, P. Chow, J. Kung, H. Liu, H. K. Mao, and R. J. Hemley, Nature (London) 436, 377 (2005).

[23] Y. Fei, L. Zhang, A. Corgne, H. Watson, A. Ricolleau, Y. Meng, and V. Prakapenka, Geophys. Res. Lett. 34, L17307 (2007).

[24] J.-F. Lin, G. Vank, S. D. Jacobsen, V. Iota, V. V. Struzhkin, V. B. Prakapenka, A. Kuznetsov, and C.-S. Yoo, Science 317, 1740 (2007). 
[25] H. Marquardt, S. Speziale, H. J. Reichmann, D. J. Frost, F. R. Schilling, and E. J. Garnero, Science 324, 224 (2009).

[26] I. Y. Kantor, L. S. Dubrovinsky, and C. A. McCammon, Phys. Rev. B 73, 100101 (2006).

[27] K. Ohta, K. Fujino, Y. Kuwayama, T. Kondo, K. Shimizu, and Y. Ohishi, J. Geophys. Res. Solid Earth 119, 4656 (2014).

[28] Z. Mao, J.-F. Lin, J. Liu, and V. B. Prakapenka, Geophys. Res. Lett. 38, L23308 (2011).

[29] N. V. Solomatova, J. M. Jackson, W. Sturhahn, J. K. Wicks, J. Zhao, T. S. Toellner, B. Kalkan, and W. M. Steinhardt, Am. Mineral. 101, 1084 (2016).

[30] S. Speziale, A. Milner, V. E. Lee, S. M. Clark, M. P. Pasternak, and R. Jeanloz, Proc. Natl. Acad. Sci. USA 102, 17918 (2005).

[31] I. S. Lyubutin, V. V. Struzhkin, A. A. Mironovich, A. G. Gavriliuk, P. G. Naumov, J.-F. Lin, S. G. Ovchinnikov, S. Sinogeikin, P. Chow, Y. Xiao et al., Proc. Natl. Acad. Sci. USA 110, 7142 (2013).

[32] R. E. Cohen, I. I. Mazin, and D. G. Isaak, Science 275, 654 (1997).

[33] D. G. Isaak, R. E. Cohen, M. J. Mehl, and D. J. Singh, Phys. Rev. B 47, 7720 (1993).

[34] I. I. Mazin and V. I. Anisimov, Phys. Rev. B 55, 12822 (1997).

[35] Z. Fang, K. Terakura, H. Sawada, T. Miyazaki, and I. Solovyev, Phys. Rev. Lett. 81, 1027 (1998).

[36] Z. Fang, I. V. Solovyev, H. Sawada, and K. Terakura, Phys. Rev. B 59, 762 (1999).

[37] S. A. Gramsch, R. E. Cohen, and S. Y. Savrasov, Am. Mineral. 88, 257 (2003).

[38] S. G. Ovchinnikov, JETP Lett. 94, 192 (2011).

[39] S. G. Ovchinnikov, T. M. Ovchinnikova, P. G. Dyad'kov, V. V. Plotkin, and K. D. Litasov, JETP Lett. 96, 129 (2012).

[40] T. Tsuchiya, R. M. Wentzcovitch, C. R. S. da Silva, and S. de Gironcoli, Phys. Rev. Lett. 96, 198501 (2006).

[41] K. Persson, A. Bengtson, G. Ceder, and D. Morgan, Geophys. Res. Lett. 33, L16306 (2006).

[42] R. M. Wentzcovitch, J. F. Justo, Z. Wu, C. R. S. da Silva, D. A. Yuen, and D. Kohlstedt, Proc. Natl. Acad. Sci. USA 106, 8447 (2009).

[43] Z. Wu, J. F. Justo, and R. M. Wentzcovitch, Phys. Rev. Lett. 110, 228501 (2013).

[44] E. Holmström and L. Stixrude, Phys. Rev. Lett. 114, 117202 (2015).

[45] Z. Wu and R. M. Wentzcovitch, Proc. Natl. Acad. Sci. USA 111, 10468 (2014).

[46] W. Metzner and D. Vollhardt, Phys. Rev. Lett. 62, 324 (1989).

[47] G. Kotliar, S. Y. Savrasov, K. Haule, V. S. Oudovenko, O. Parcollet, and C. A. Marianetti, Rev. Mod. Phys. 78, 865 (2006).

[48] A. Georges, G. Kotliar, W. Krauth, and M. J. Rozenberg, Rev. Mod. Phys. 68, 13 (1996).

[49] G. Kotliar and D. Vollhardt, Phys. Today 57(3), 53 (2004).

[50] K. Haule, Phys. Rev. B 75, 155113 (2007).

[51] L. V. Pourovskii, B. Amadon, S. Biermann, and A. Georges, Phys. Rev. B 76, 235101 (2007).

[52] B. Amadon, F. Lechermann, A. Georges, F. Jollet, T. O. Wehling, and A. I. Lichtenstein, Phys. Rev. B 77, 205112 (2008).

[53] H. Park, A. J. Millis, and C. A. Marianetti, Phys. Rev. B 90, 235103 (2014).

[54] S. Y. Savrasov, G. Kotliar, and E. E. Abrahams, Nature (London) 410, 793 (2001).
[55] G. Keller, K. Held, V. Eyert, D. Vollhardt, and V. I. Anisimov, Phys. Rev. B 70, 205116 (2004).

[56] J. Kuneš, A. V. Lukoyanov, V. I. Anisimov, R. T. Scalettar, and W. E. Pickett, Nat. Mater. 7, 198 (2008).

[57] J. Kuneš, V. I. Anisimov, S. L. Skornyakov, A. V. Lukoyanov, and D. Vollhardt, Phys. Rev. Lett. 99, 156404 (2007).

[58] J. Kuneš, D. M. Korotin, M. A. Korotin, V. I. Anisimov, and P. Werner, Phys. Rev. Lett. 102, 146402 (2009).

[59] I. Leonov, A. I. Poteryaev, V. I. Anisimov, and D. Vollhardt, Phys. Rev. Lett. 106, 106405 (2011).

[60] N. A. Skorikov, A. O. Shorikov, S. L. Skornyakov, M. A. Korotin, and V. I. Anisimov, J. Phys.: Condens. Matter 27, 275501 (2015).

[61] I. Leonov, D. Korotin, N. Binggeli, V. I. Anisimov, and D. Vollhardt, Phys. Rev. B 81, 075109 (2010).

[62] A. O. Shorikov, Z. V. Pchelkina, V. I. Anisimov, S. L. Skornyakov, and M. A. Korotin, Phys. Rev. B 82, 195101 (2010).

[63] I. Leonov, A. I. Poteryaev, V. I. Anisimov, and D. Vollhardt, Phys. Rev. B 85, 020401 (2012).

[64] V. Křápek, P. Novák, J. Kuneš, D. Novoselov, D. M. Korotin, and V. I. Anisimov, Phys. Rev. B 86, 195104 (2012).

[65] L. V. Pourovskii, J. Mravlje, M. Ferrero, O. Parcollet, and I. A. Abrikosov, Phys. Rev. B 90, 155120 (2014).

[66] P. Delange, T. Ayral, S. I. Simak, M. Ferrero, O. Parcollet, S. Biermann, and L. Pourovskii, Phys. Rev. B 94, 100102 (2016).

[67] D. Grieger, C. Piefke, O. E. Peil, and F. Lechermann, Phys. Rev. B 86, 155121 (2012).

[68] I. Leonov, V. I. Anisimov, and D. Vollhardt, Phys. Rev. B 91, 195115 (2015).

[69] I. Leonov, Phys. Rev. B 92, 085142 (2015).

[70] I. Leonov, L. Pourovskii, A. Georges, and I. A. Abrikosov, Phys. Rev. B 94, 155135 (2016).

[71] I. Leonov, E. Greenberg, M. P. Belov, G. K. Rozenberg, and I. A. Abrikosov, High Pressure Res. 37, 96 (2017).

[72] C. D. Lorenz and R. M. Ziff, Phys. Rev. E 57, 230 (1998).

[73] In our calculations, we do not take into account a possible decomposition of $(\mathrm{Fe}, \mathrm{Mg}) \mathrm{O}$ under high-pressure, hightemperature conditions, as proposed in Ref. [90].

[74] We note that by construction this procedure preserves the fcc symmetry of the unit cell. In the so-constructed supercells the underlying cubic symmetry turned out to be preserved, which allows us to use the cubic Brillouin zone for further analysis of the electronic structure.

[75] I. Kantor, L. Dubrovinsky, C. McCammon, G. Steinle-Neumann, A. Kantor, N. Skorodumova, S. Pascarelli, and G. Aquilanti, Phys. Rev. B 80, 014204 (2009).

[76] E. Greenberg, I. Leonov, S. Layek, Z. Konopkova, M. P. Pasternak, L. Dubrovinsky, R. Jeanloz, I. A. Abrikosov, and G. K. Rozenberg, arXiv:1706.02787.

[77] F. Birch, Phys. Rev. 71, 809 (1947).

[78] S. Baroni, S. de Gironcoli, A. Dal Corso, and P. Giannozzi, Rev. Mod. Phys. 73, 515 (2001).

[79] P. Giannozzi, S. Baroni, N. Bonini, M. Calandra, R. Car, C. Cavazzoni, D. Ceresoli, G. L. Chiarotti, M. Cococcioni, I. Dabo et al., J. Phys.: Condens. Matter 21, 395502 (2009).

[80] N. Marzari and D. Vanderbilt, Phys. Rev. B 56, 12847 (1997).

[81] N. Marzari, A. A. Mostofi, J. R. Yates, I. Souza, and D. Vanderbilt, Rev. Mod. Phys. 84, 1419 (2012). 
[82] V. I. Anisimov, D. E. Kondakov, A. V. Kozhevnikov, I. A. Nekrasov, Z. V. Pchelkina, J. W. Allen, S.-K. Mo, H.-D. Kim, P. Metcalf, S. Suga et al., Phys. Rev. B 71, 125119 (2005).

[83] G. Trimarchi, I. Leonov, N. Binggeli, D. Korotin, and V. I. Anisimov, J. Phys.: Condens. Matter 20, 135227 (2008).

[84] D. Korotin, A. V. Kozhevnikov, S. L. Skornyakov, I. Leonov, N. Binggeli, V. I. Anisimov, and G. Trimarchi, Eur. Phys. J. B 65, 91 (2008).

[85] A. V. Ruban and I. A. Abrikosov, Rep. Prog. Phys. 71, 046501 (2008).

[86] E. Gull, A. J. Millis, A. I. Lichtenstein, A. N. Rubtsov, M. Troyer, and P. Werner, Rev. Mod. Phys. 83, 349 (2011).
[87] Interestingly, for the LS state the estimated value of $K_{0, T}$ depends very sensitively on the details of the total-energy fitting, e.g., on the choice of the LS region. This can lead to a sufficient underestimation of $K_{0, T}$; for example, in previous reports [69-71] it is suggested that $K_{0, T} \sim 162 \mathrm{GPa}$.

[88] J.-F. Lin, A. G. Gavriliuk, V. V. Struzhkin, S. D. Jacobsen, W. Sturhahn, M. Y. Hu, P. Chow, and C.-S. Yoo, Phys. Rev. B 73, 113107 (2006).

[89] J. Kuneš and V. Křápek, Phys. Rev. Lett. 106, 256401 (2011).

[90] L. S. Dubrovinsky, N. A. Dubrovinskaia, S. K. Saxena, H. Annersten, E. Hålenius, H. Harryson, F. Tutti, S. Rekhi, and T. L. Bihan, Science 289, 430 (2000). 